OPEN ACCESS

Edited by:

Jean-Paul R-Richalet, Université Paris 13, France

Reviewed by: Akira Yoshii,

University of Illinois at Chicago,

United States

Melissa L. Bates,

University of lowa, United States

${ }^{*}$ Correspondence:

Patrick Müller

patrick.mueller@dzne.de

${ }^{\dagger}$ These authors have contributed equally to this work

Specialty section:

This article was submitted to Integrative Physiology, a section of the journal Frontiers in Physiology

Received: 18 June 2018 Accepted: 04 September 2018 Published: 01 October 2018

Citation: Becke A, Müller P, Dordevic M,

Lessmann V, Brigadski $T$ and Müller NG (2018) Daily Intermittent Normobaric Hypoxia Over 2 Weeks

Reduces BDNF Plasma Levels in Young Adults - A Randomized

Controlled Feasibility Study.

Front. Physiol. 9:1337.

doi: 10.3389/fphys.2018.01337

\section{Daily Intermittent Normobaric Hypoxia Over 2 Weeks Reduces BDNF Plasma Levels in Young Adults - A Randomized Controlled Feasibility Study}

\author{
Andreas Becke ${ }^{1,2+}$, Patrick Müller ${ }^{2 * t}$, Milos Dordevic², Volkmar Lessmann ${ }^{3,4}$, \\ Tanja Brigadski ${ }^{3,5}$ and Notger G. Müller ${ }^{2,4}$
}

1 Institute of Cognitive Neurology and Dementia Research, Otto-von-Guericke-Universität Magdeburg, Magdeburg, Germany, ${ }^{2}$ Neuroprotection Laboratory, German Center for Neurodegenerative Diseases (DZNE), Magdeburg, Germany, ${ }^{3}$ Institute of Physiology, Otto-von-Guericke-Universität Magdeburg, Magdeburg, Germany, ${ }^{4}$ Center for Behavioral Brain Sciences, Magdeburg, Germany, ${ }^{5}$ Informatics and Microsystem Technology, University of Applied Sciences, Kaiserslautern, Kaiserslautern, Germany

Background: The results from animal and human research indicate that acute intermittent hypoxia can enhance brain-derived neurotrophic factor (BDNF) plasma levels and gene expression. As BDNF is known to promote the differentiation of new neurons and the formation of synapses, it has been proposed to mediate adult neuroplasticity. Thus, the present study aimed to analyze the long-term effects of daily intermittent exposure to normobaric hypoxia (simulating high altitude exposure at approximately 4000-5000 m) over 2 weeks on BDNF levels in young adults.

Methods: Twenty-eight young adults (age: 19-33 years) were randomized into a hypoxic intervention group ( $N=14)$ or the control group $(N=14)$. Participants in the intervention group breathed intermittent normobaric hypoxic air at resting conditions (5 min intervals, 80-85\% $\mathrm{SpO}_{2}$ measured via a finger pulse oximeter, 12 sessions for $60 \mathrm{~min} /$ day for 2 weeks) via a hypoxic generator. BDNF plasma and serum levels were determined at baseline and at 2 weeks after intervention using sandwich ELISAs.

Results: After 2 weeks of daily intermittent hypoxic treatment $(\mathrm{IHT})$, we found a significant group $\mathrm{x}$ time interaction effect for BDNF plasma levels based on a significant decrease in BDNF levels in the hypoxia group.

Conclusion: Our results demonstrate that daily intermittent administration of hypoxic air has a significant effect on BDNF regulation in healthy young adults. Contrary to other results reporting an increase in BDNF levels under hypoxic conditions, the present data suggest that hypoxic treatment using intensive $\mathrm{IHT}$ can reduce BDNF plasma levels for at least 2 weeks. This finding indicates that the daily application of hypoxic air is too frequent for the aimed physiological response, namely, an increase in BDNF levels.

\footnotetext{
Keywords: hypoxia, BDNF, neuroplasticity, IHT, adaptation
} 


\section{INTRODUCTION}

Hypoxia is defined by a reduced oxygen content in air and can be divided into intermittent and chronic forms. Thereby, intermittent hypoxia applies to a large spectrum of stimuli that range from exercise in high altitude to obstructive sleep apnea (OSA). Intermittent hypoxia treatment (IHT) was first used in sports medicine to enhance human physical performance (erythropoiesis and angiogenesis) (Viscor et al., 2018). During the following years, hypoxic training was increasingly employed for non-pharmacological treatment of several diseases (e.g., bronchial asthma, hypertension, and cardiovascular diseases). IHT can effectively stimulate various metabolic processes (Serebrovskaya et al., 2008) and can have numerous positive health effects similar to cardiovascular physical activity (Enette et al., 2017). IHT may serve as a protective mechanism for the brain by inducing neurogenesis. For instance, histological studies in adult rats have shown that IHT promotes a transient increase in progenitor cell proliferation in the subventricular zone and a long-term increase in the dentate gyrus (Zhu et al., 2005) and has the potential to recover spatial learning deficits after cerebral ischemia by increased hippocampal neurogenesis (Tsai et al., 2011). However, intermittent normobaric hypoxia is not associated with positive effects only per se. For example, the clinical syndrome of OSA leads to intermittent hypoxia as well (Burtscher et al., 2009) and is associated with numerous negative effects such as reduced cognitive performance (Yan, 2014; Malle et al., 2016). Hence, based on different characteristics such as the dose and the duration, we can assume that hypoxia induces both protective and pathological effects. It has been proposed that low-dose intermittent hypoxia (9-16\% inspired $\mathrm{O}_{2}$ ) with short durations can enhance positive physiological processes, whereby high-dose hypoxia $\left(2-8 \%\right.$ inspired $\left.\mathrm{O}_{2}\right)$ is associated with progressively pathological mechanisms (Navarrete-Opazo and Mitchell, 2014).

The results from animal and human research indicate that acute intermittent hypoxia (Vermehren-Schmaedick et al., 2012) and physical activity (Enette et al., 2017) can enhance brainderived neurotrophic factor (BDNF) blood levels and BDNF gene expression. Such gene expression is explained by an oxygen deficit recognized by the oxygen sensory system (Sharp and Bernaudin, 2004) changing the oxygen-dependent degradation domain of hypoxia-inducible factor (HIF-1), thereby inducing an increase in HIF-1-alpha levels (Wiener et al., 1996). HIF1-alpha is known to act as a transcription factor to modulate the expression of several genes, such as BDNF growth factor levels (Helan et al., 2014). The BDNF neurotrophin is a member of the nerve growth factor family and is widely expressed in the human brain, especially in the hippocampus, but it is also expressed in peripheral tissues such as the pulmonary vasculature (Aravamudan et al., 2012; Helan et al., 2014). Current research studies indicate BNDF plasma levels as a potential biomarker for reliable diagnosis of neurocognitive disorders (Levada et al., 2016). The protein is secreted in an activitydependent manner but is also secreted in response to hypoxia (Haubensak et al., 1998; Hartmann et al., 2001; Kohara et al., 2001; Brigadski et al., 2005; Matsuda et al., 2009; Brigadski and Leßmann, 2014; Helan et al., 2014; Edelmann et al., 2015; Hartman et al., 2015). Research results indicate that $75 \%$ of the BDNF in the peripheral blood plasma originates from the brain (Krabbe et al., 2007; Rasmussen et al., 2009). Several studies have suggested that BDNF is an important modulator of the CNS and promotes the differentiation of new neurons and synapses (Huang and Reichardt, 2001; Leschik et al., 2013; Park and Poo, 2013; Edelmann et al., 2014). BDNF, therefore, represents one of the major mediators of neuroplasticity (Calabrese et al., 2014). Furthermore, some authors have suggested that BDNF blood levels may serve as a biomarker for the diagnosis of neurodegenerative diseases and psychiatric disorders and can also serve as a surrogate marker for the success of therapies in these disorders (Ruscheweyh et al., 2011). Reduced BDNF blood levels have been reported in Alzheimer's disease (Laske et al., 2007) and mild cognitive impairment (Forlenza et al., 2010).

Regarding the effect of intermittent hypoxia on BDNF blood levels in humans, the status of research is currently unclear. The results from animal and human studies have shown an acute increase in BDNF plasma levels in response to hypoxia. Helan et al. (2014) observed an increase in BDNF levels in 30 healthy volunteers after $72 \mathrm{~h}$ of normobaric hypoxia. Schega et al. (2016) reported no effects on BDNF in serum in older adults $(N=34,66.4 \pm 3.3$ years $)$ after 4 weeks of intermittent normobaric hypoxia $(3 \times$ per week for $90 \mathrm{~min}$ ) in addition to cardiovascular exercise. However, their data indicated that BDNF levels increased in the exercise-intervention group and in the exercise control-group after a compensation period of several weeks. This finding raises the question of whether the delayed effect could have been observed after hypoxic treatment alone, i.e., without concomitant cardiovascular exercise intervention.

Previous studies in animal research indicate an occurrence of neurogenesis in dentate gyrus within 4 weeks subsequent to intermittent hypoxia (Zhu et al., 2005). Based on these results we conducted a feasibility study to test the effects of 2 weeks of daily exposure to hypoxic air, which simulated intermittent hypoxia treatment (IHT), on peripheral BDNF levels. Therefore, we expected an increase in BDNF levels (as a central mediator of neurogenesis).

With respect to previous research on passive IHT methods, a protocol was chosen that has been shown to increase aerobic capacity and exercise tolerance in elderly men (Burtscher et al., 2004). In view of the data from Zhu et al. (2005) and based on recommendations for IHT regimes (Bassovitch and Serebrovskaya, 2009), we estimated the peak long-term effects of IHT to emerge 2 weeks after the intervention. If successful, this process is an easy to administer, low-cost intervention that may have great potential in inducing neuroplasticity and preventing cognitive deficits.

\section{MATERIALS AND METHODS}

The study was designed as a two-week randomized, controlled intervention. The ethics committee at the Otto-von-GuerickeUniversität Magdeburg, approved the study, and all of the subjects signed a written informed consent form prior to 
participation. The exclusion criteria were acute or chronic cardiovascular, renal, metabolic, orthopedic and/or neurological diseases.

Twenty-eight young adults (age: 19-33 years) were randomized to a hypoxic intervention group $[N=14$ (9 female), mean age 27.78, $S D=2.39$ ] or a control group $[N=14$ (5 female), mean age 22.85, SD 2.35] using the website www.randomization.com. The participants in the intervention group breathed intermittent normobaric hypoxic air at resting conditions (5 min intervals at a target of $80-85 \% \mathrm{SpO}_{2}$ via a finger pulse oximeter, 12 sessions for $60 \mathrm{~min} /$ day for 2 weeks) generated by a hypoxic generator (b-cat and integra ten). The simulated high altitude was continuously manually adjusted between 4000 and $5000 \mathrm{~m}$ to reach the target $\mathrm{SpO}_{2}$. The control group received no intervention.

Fasting blood samples were taken in the mornings at baseline and at posttest ( 2 weeks after the last training session). From the blood samples, the plasma and serum concentrations of BDNF were determined using sandwich ELISAs (BDNF DuoSet; R\&D Systems, Wiesbaden, Germany) as previously described (Schega et al., 2016).

For the intervention group, the blood samples for the small blood count were taken 4 times at baseline, 1 week after the intervention, at the end of intervention (consecutive day of last intervention session) and 2 weeks after the intervention. Five missing data sets for the second time point and 3 missing data sets for the third time point were reported (subjects did not show up).

Statistical analysis of BDNF plasma levels, BDNF serum levels and small blood count levels were performed with SPSS (SPSS 22 Inc./IBM). The intervention effects for BDNF were tested using repeated-measures ANOVAs with group (IHT and CG) as the between-subject factor and time (pre and post) as the withinsubject factor. Age and gender were included as covariates. Additionally, post hoc pairwise comparisons were performed to determine the longitudinal changes in the hypoxia and control groups separately. In the case of non-normal distribution of data, we used the Mann-Whitney U-test or the Wilcoxon test instead of $t$-tests. The effect size was quantified by partial eta squared $\left(\eta^{2}\right)$. For interaction effects, the percentage changes from baseline to post measures were calculated for BDNF and small blood count values and were then correlated with Pearson's formula.

\section{RESULTS}

The plasma and serum levels of BDNF were analyzed in the blood samples before the onset of the intervention as well as after the intervention. A significant group $\mathrm{x}$ time interaction effect was observed for the BDNF plasma levels $[F(1,26)=10.742, p=0.002$, $\left.\eta^{2}=0.292\right]$. Post hoc pairwise comparisons showed a significant decrease in BDNF plasma levels only in the hypoxia group from baseline to the posttest period (Wilcoxon-Test, $Z=-3.296$, $p=0.001$ ). The intraindividual changes in BDNF plasma levels reached a reduction of $66.34 \%$ of the pretreatment period. No significant time $\times$ group interactions emerged for BDNF serum levels (see Table 1 and Figure 1).
For the intervention group, blood samples for a small blood count were collected 4 times at baseline, 1 week after the intervention, at the end of the intervention (consecutive day of the last intervention session) and 2 weeks after the intervention. Five missing data sets for the second time point and 3 missing data sets for the third time point were reported (subjects did not show up). Using mixed linear effects to model the effect over time, the red blood cell distribution showed a linear decrease over time $(p<0.01$; Table 2).

Furthermore, an analysis of Pearson correlations between the baseline to post measure changes (\%) revealed a close to significant positive correlation (one-tailed) for BDNF plasma and leucocyte counts $\left(\mathrm{R}_{\mathrm{WBC}}=0.446 ; p=0.055\right)$ and a trend for a negative $B D N F$ and lymphocyte interaction $\left(\mathrm{R}_{\mathrm{lym}}=-0.374\right.$; $p=0.094)$.

\section{DISCUSSION}

Normobaric hypoxia such as with high-altitude training is generally assumed to have positive effects on physical and cognitive performance. Here, we tested the effect of a daily intermittent normobaric hypoxic training during a period of 2 weeks on the BDNF levels. While we observed the expected effects on blood parameters such as on the mean corpuscular hemoglobin concentration, contrary to our expectation, we found BDNF plasma levels to be significantly reduced 2 weeks after daily intermittent normobaric hypoxia over a period of 2 weeks. Regarding BDNF serum levels, no changes were detected. Research results from Pan et al. (1998) indicate that BDNF can pass the blood brain barrier by a high-capacity, saturable transport system and that 75\% of BDNF plasma levels stems from the brain (Krabbe et al., 2007; Rasmussen et al., 2009).

Decreased BDNF levels are typically found in animal research when the animals have previously experienced stress. Various types of stress, including oxidative stress, have been shown to lead to decreased BDNF gene expression in cortical regions, including the hippocampus (Smith, 1996; Smith and Cizza, 1996; Bath et al., 2013; Kwon et al., 2013; Rothman and Mattson, 2013). In humans, a reduction in BDNF levels was seen after muscle damage or with very intensive physical exercise. To avoid such overtraining, successful exercise training is known to require sufficient resting periods (Parra et al., 2000). In rodents, physical activity induces BDNF gene expression in cortical regions, especially in the hippocampus (Neeper et al., 1995; Uysal et al., 2015). Studies on humans have reported an increase in BDNF levels following sportive interventions (Erickson et al., 2012; Müller et al., 2017a,b; Rehfeld et al., 2018). Others, however, failed to show changes in the levels of any of the neurotrophic factors that were assessed (Maass et al., 2016). A current review by Enette et al. (2017) provides a comprehensive analysis of the effects of aerobic training on BDNF plasma and serum levels in older adults. In 11 of the 14 randomized controlled trials that were included, the authors reported significantly increased BDNF plasma and/or serum levels after aerobic intervention.

Together, these findings indicate that our IHT protocol with its daily applications of hypoxic air might have been too intensive 
TABLE 1 | Statistics of rANOVA on BDNF plasma and serum levels.

\begin{tabular}{|c|c|c|c|c|c|c|c|c|c|c|}
\hline \multirow[b]{2}{*}{ BDNF } & \multirow[b]{2}{*}{$d f$} & \multicolumn{3}{|c|}{ Time } & \multicolumn{3}{|c|}{ Group } & \multicolumn{3}{|c|}{ Interaction (time $x$ group) } \\
\hline & & $\boldsymbol{F}$ & $P$ & $\eta^{2}$ & $\boldsymbol{F}$ & $p$ & $\eta^{2}$ & $\boldsymbol{F}$ & $p$ & $\eta^{2}$ \\
\hline plasma level & 1,26 & 11.52 & $0.002^{* *}$ & 0.307 & 0.425 & 0.520 & 0.016 & 10.742 & $0.003^{* *}$ & 0.292 \\
\hline Serum level & 1,25 & 2.24 & 0.147 & 0.082 & 10.53 & $0.003^{* *}$ & 0.296 & 3.68 & 0.066 & 0.128 \\
\hline
\end{tabular}

$* * p<0.01$.
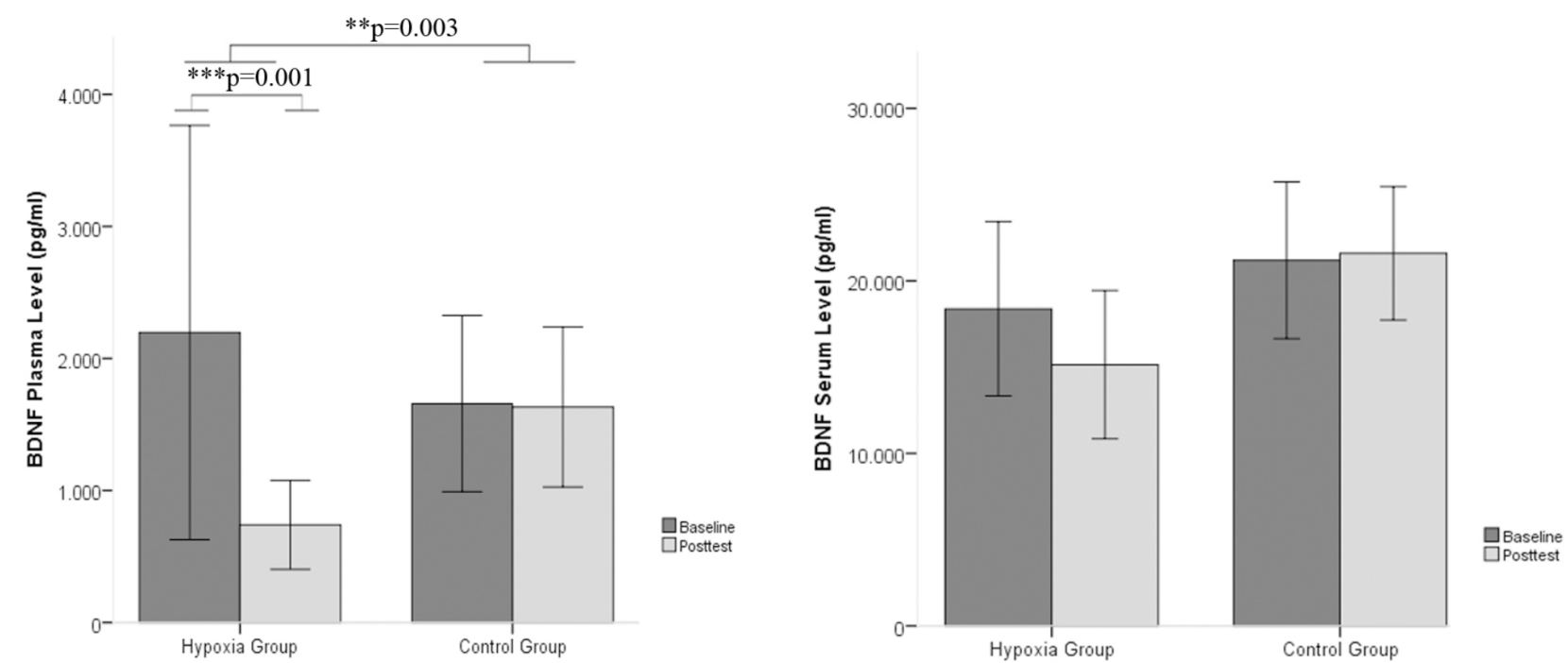

FIGURE 1 | BDNF plasma and serum levels between baseline and post intervention measures in the hypoxia intervention group and the control group (Mean \pm SD). $* * p<0.01$ and ${ }^{* * *} p<0.001$.

TABLE 2 | Small blood count for the treatment group.

\section{Small blood count}

\begin{tabular}{|c|c|c|c|c|c|c|c|c|c|c|c|c|}
\hline & \multicolumn{2}{|c|}{ Baseline $(N=14)$} & \multicolumn{2}{|c|}{1 week IHT $(N=9)$} & \multicolumn{2}{|c|}{2 weeks IHT $(N=11)$} & \multicolumn{2}{|c|}{ Posttest $(N=14)$} & \multicolumn{2}{|c|}{ Linear time effect } & \multicolumn{2}{|c|}{ Quadratic time effect } \\
\hline & Mean & SD & Mean & SD & Mean & SD & Mean & SD & $\boldsymbol{F}$ & $p$ & $\boldsymbol{F}$ & $p$ \\
\hline $\operatorname{RBCC}\left(\times 10^{6} / \mathrm{ml}\right)$ & 4.94 & 0.43 & 5.00 & 0.29 & 4.75 & 0.36 & 4.83 & 0.36 & 1.066 & 0.308 & 0.628 & 0.539 \\
\hline RDW (\%) & 13.66 & 0.90 & 13.64 & 0.13 & 12.70 & 0.62 & 12.61 & 0.59 & 20.84 & $0.00 * *$ & 1.259 & 0.295 \\
\hline $\mathrm{HCT}$ & 0.46 & 0.03 & 0.44 & 0.03 & 0.43 & 0.03 & 0.45 & 0.03 & 1.852 & 0.181 & 1.443 & 0.248 \\
\hline $\mathrm{HBG}(\mathrm{mmol} / \mathrm{L})$ & 9.07 & 0.72 & 9.22 & 0.81 & 8.89 & 0.62 & 8.74 & 0.58 & 2.120 & 0.153 & 0.318 & 0.729 \\
\hline $\mathrm{MCH}$ (fmol) & 1.84 & 0.13 & 1.84 & 0.08 & 1.87 & 0.10 & 1.81 & 0.09 & 0.287 & 0.595 & 0.903 & 0.414 \\
\hline $\mathrm{MCHC}(\mathrm{mmol} / \mathrm{l})$ & 19.79 & 0.79 & 21.02 & 0.55 & 20.48 & 0.52 & 19.70 & 0.53 & 0.163 & 0.689 & 11.77 & $0.000 * *$ \\
\hline MCV (fl) & 93.14 & 3.80 & 87.40 & 2.79 & 91.64 & 4.37 & 92.07 & 4.08 & 0.145 & 0.705 & 3.866 & $0.029 *$ \\
\hline WBC $\left(\times 10^{3} / \mathrm{ml}\right)$ & 8.22 & 3.72 & 7.72 & 1.80 & 7.28 & 0.75 & 6.21 & 0.80 & 5.099 & $0.030 *$ & 0.106 & 0.899 \\
\hline SumGranul & 0.63 & 0.10 & 0.66 & 0.06 & 0.61 & 0.06 & 0.58 & 0.06 & 3.369 & $0.074^{t}$ & 0.603 & 0.552 \\
\hline Segs & 0.60 & 0.10 & 0.63 & 0.05 & 0.58 & 0.07 & 0.55 & 0.06 & 3.839 & $0.057^{t}$ & 0.908 & 0.411 \\
\hline Mono & 0.06 & 0.01 & 0.07 & 0.01 & 0.06 & 0.02 & 0.06 & 0.02 & 0.071 & 0.792 & 0.496 & 0.613 \\
\hline Thrombocyte (×103/ml) & 285.14 & 78.16 & 278.80 & 41.56 & 271.91 & 66.43 & 252.64 & 42.76 & 1.883 & 0.178 & 0.074 & 0.929 \\
\hline
\end{tabular}

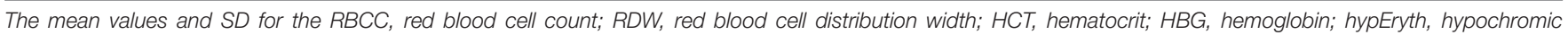

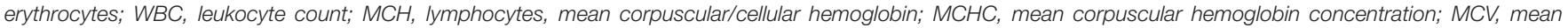

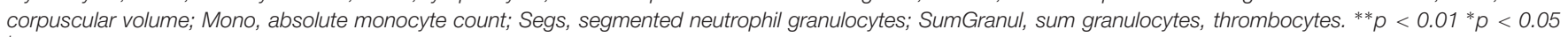
${ }^{t} p<0.09$.

and, therefore, too stressful for the participants' bodies. In agreement with this finding, we observed a change in blood marker levels that were indicative of inflammation, namely, lymphocytes and granulocytes. Intensive physical exercise also induces inflammatory processes (Brown et al., 2015), and the latter has also been shown to relate to reduced BDNF levels after 
acute exercise at higher intensities (Nofuji et al., 2012; CabralSantos et al., 2016). Other conditions in which a reduction in BDNF levels was observed in the past include sleep apnea (Wang et al., 2012), birth stress associated with psychiatric disease later in life (Cannon et al., 2008), and stroke with low functional outcome (Lasek-Bal et al., 2015). With respect to the present study, the results of Wang et al. (2017) are of special relevance, as sleep apnea is associated with nocturnal intermittent hypoxia. Again, this finding suggests that "overdosing" hypoxia has detrimental effects on BDNF secretion.

The assumption that our IHT protocol was too intense and therefore decreased BDNF levels leads to the crucial question of whether other less stressful IHT protocols could still have a positive effect. In addition, methodological aspects (sampling time and preanalytical variations) could have an influence on the gained results. Indeed, there is an ongoing discussion of what type of hypoxia treatment is most effective (Serebrovskaya and Xi, 2016). A protocol that increases physical fitness at the same time may have negative effects on BDNF (Enette et al., 2017). Indeed, we had used a protocol that, in a former study, had shown positive effects on aerobic capacity.

\section{Metabolic and Cardiovascular Response to Hypoxia}

Several field experiments in the mountains and environmental studies in chambers report physiological effects of hypoxia (Heinonen et al., 2016). These experiments show that hypoxia can induce cardiovascular stress, can increase sympathetic neural activation and can alter energy metabolism. The complex metabolic response causes a release of various stress hormones (Kayser and Verges, 2013). Regarding cardiovascular response to normobaric hypoxia Heinonen et al. (2014) reported a significantly increased cardiac output, ejection fraction and tachycardia. Additionally, Heinonen et al. (2014, 2017) discuss hypoxia as a potential trigger for the release of brain natriuretic peptide (BNP) and the hormone apelin.

\section{Limitations and Outlook}

This randomized controlled feasibility study has several limitations. First, the sample size was small $(N=28)$. Second, the

\section{REFERENCES}

Aravamudan, B., Thompson, M., Pabelick, C., and Prakash, Y. S. (2012). Brainderived neurotrophic factor induces proliferation of human airway smooth muscle cells. J. Cell Mol. Med. 16, 812-823. doi: 10.1111/j.1582-4934.2011. 01356.x

Bassovitch, O., and Serebrovskaya, T. V. (2009). "Equipment and regimes for intermittent hypoxia therapy," in Intermittent Hypoxia: From Molecular Mechanismsto Clinical Applications, eds L. Xi and T. V. Serebrovskaya (New York, NY: Nova Science Publishers).

Bath, K. G., Schilit, A., and Lee, F. S. (2013). Stress effects on BDNF expression: effects of age, sex, and form of stress. Neuroscience 239, 149-156. doi: 10.1016/j. neuroscience.2013.01.074

Brigadski, T., Hartmann, M., and Lessmann, V. (2005). Differential vesicular targeting and time course of synaptic secretion of the mammalian neurotrophins. J. Neurosci. 25, 7601-7614. doi: 10.1523/JNEUROSCI.1776-05. 2005 blood samples were only analyzed at baseline and after 2 weeks of intervention. Another limiting factor in the BDNF blood analyses is the large variances.

Future studies are needed to evaluate the correct dose of normobaric intermittent hypoxia to increase BDNF plasma levels and examine the underlying neurobiological mechanisms. An intensive assessment (neuropsychology, MRI/PET, cortisol, and IGF-1) would be useful to analyze the physiological adaptations to hypoxia.

In addition, BDNF has been suggested to play a mediating role in schizophrenia (Sokoloff et al., 2004; Guillin et al., 2007). Thus, several studies indicate an increase of the BDNF levels and gene expression in patients with schizophrenia (Laske and Eschweiler, 2006). In conclusion, an intermittent normobaric hypoxia regimen that successfully increases the BDNF levels may offer a non-pharmacological treatment to patients with schizophrenia.

\section{ETHICS STATEMENT}

This study was carried out in accordance with the recommendations of Ethics Committee of the Medical Faculty at the Otto-von-Guericke-Universität Magdeburg with written informed consent from all subjects. All subjects gave written informed consent in accordance with the Declaration of Helsinki. The protocol was approved by the Ethics Committee of the Medical Faculty at the Otto-von-Guericke-Universität Magdeburg.

\section{AUTHOR CONTRIBUTIONS}

AB contributed to study organization, data analysis, paper writing, and paper reviewing. PM contributed to data analysis, paper writing, and paper reviewing. MD reviewed the paper. VL and TB contributed to data analysis and paper reviewing. NM contributed to study organization, paper writing, and paper reviewing.

Brigadski, T., and Leßmann, V. (2014). BDNF: a regulator of learning and memory processes with clinical potential. e-Neuroforum 5, 1-11. doi: 10.1007/s13295014-0053-9

Brown, W. M., Davison, G. W., McClean, C. M., and Murphy, M. H. (2015). A systematic review of the acute effects of exercise on immune and inflammatory indices in untrained adults. Sports Med. Open 1:35. doi: 10.1186/ s40798-015-0032-x

Burtscher, M., Haider, T., Domej, W., Linser, T., Gatterer, H., and Faulhaber, M. (2009). Intermittent hypoxia increases exercise tolerance in patients at risk for or with mild COPD. Respir. Physiol. Neurobiol. 165, 97-103. doi: 10.1016/j.resp. 2008.10.012

Burtscher, M., Pachinger, O., Ehrenbourg, I., Mitterbauer, G., Faulhaber, M., Puhringer, R., et al. (2004). Intermittent hypoxia increases exercise tolerance in elderly men with and without coronary artery disease. Int. J. Cardiol. 96, 247-254. doi: 10.1016/j.ijcard.2003.07.021

Cabral-Santos, C., Castrillon, C. I., Miranda, R. A., Monteiro, P. A., Inoue, D. S., Campos, E. Z., et al. (2016). Inflammatory cytokines and BDNF response to 
high-intensity intermittent exercise: effect the exercise volume. Front. Physiol. 7:509. doi: 10.3389/fphys.2016.00509

Calabrese, F., Rossetti, A. C., Racagni, G., Gass, P., Riva, M. A., and Molteni, R. (2014). Brain-derived neurotrophic factor: a bridge between inflammation and neuroplasticity. Front. Cell Neurosci. 8:430. doi: 10.3389/fncel.2014.00430

Cannon, T. D., Yolken, R., Buka, S., Torrey, E. F., and Collaborative Study Group on the Perinatal Origins of Severe Psychiatric Disorders (2008). Decreased neurotrophic response to birth hypoxia in the etiology of schizophrenia. Biol. Psychiatry 64, 797-802. doi: 10.1016/j.biopsych.2008.04.012

Edelmann, E., Cepeda-Prado, E., Franck, M., Lichtenecker, P., Brigadski, T., and Lessmann, V. (2015). Theta burst firing recruits BDNF release and signaling in postsynaptic CA1 neurons in spike-timing-dependent LTP. Neuron 86, 1041-1054. doi: 10.1016/j.neuron.2015.04.007

Edelmann, E., Lessmann, V., and Brigadski, T. (2014). Pre- and postsynaptic twists in BDNF secretion and action in synaptic plasticity. Neuropharmacology 76(Pt C), 610-627. doi: 10.1016/j.neuropharm.2013.05.043

Enette, L., Vogel, T., Fanon, J. L., and Lang, P. O. (2017). Effect of interval and continuous aerobic training on basal serum and plasma brain-derived neurotrophic factor values in seniors: a systematic review of intervention studies. Rejuvenation Res. 20, 473-483. doi: 10.1089/rej.2016.1886

Erickson, K. I., Miller, D. L., and Roecklein, K. A. (2012). The aging hippocampus: interactions between exercise, depression, and BDNF. Neuroscientist 18, 82-97. doi: $10.1177 / 1073858410397054$

Forlenza, O. V., Diniz, B. S., Teixeira, A. L., Ojopi, E. B., Talib, L. L., Mendonca, V. A., et al. (2010). Effect of brain-derived neurotrophic factor Val66Met polymorphism and serum levels on the progression of mild cognitive impairment. World J. Biol. Psychiatry 11, 774-780. doi: 10.3109/ 15622971003797241

Guillin, O., Demily, C., and Thibaut, F. (2007). Brain-derived neurotrophic factor in schizophrenia and its relation with dopamine. Int. Rev. Neurobiol. 78, 377-395. doi: 10.1016/S0074-7742(06)78012-6

Hartman, W., Helan, M., Smelter, D., Sathish, V., Thompson, M., Pabelick, C. M., et al. (2015). Role of hypoxia-induced brain derived neurotrophic factor in human pulmonary artery smooth muscle. PLoS One 10:e0129489. doi: 10.1371/ journal.pone.0129489

Hartmann, M., Heumann, R., and Lessmann, V. (2001). Synaptic secretion of BDNF after high-frequency stimulation of glutamatergic synapses. EMBO J. 20, 5887-5897. doi: 10.1093/emboj/20.21.5887

Haubensak, W., Narz, F., Heumann, R., and Lessmann, V. (1998). BDNF-GFP containing secretory granules are localized in the vicinity of synaptic junctions of cultured cortical neurons. J. Cell Sci. 111(Pt 11), 1483-1493.

Heinonen, I., Luotolahti, M., Vuolteenaho, O., Nikinmaa, M., Saraste, A., Hartiala, J., et al. (2014). Circulating N-terminal brain natriuretic peptide, and cardiac function in response to acute systemic hypoxia in healthy humans. J. Transl. Med. 12:189. doi: 10.1186/1479-5876-12-189

Heinonen, I., Vuolteenaho, O., Koskenvuo, J., Arjamaa, O., and Nikinmaa, M. (2017). Systemic hypoxia increases circulating concentration of apelin in humans. High Alt. Med. Biol. 18, 292-295. doi: 10.1089/ham.2017.0017

Heinonen, I. H., Boushel, R., and Kalliokoski, K. K. (2016). The circulatory and metabolic responses to hypoxia in humans - with special reference to adipose tissue physiology and obesity. Front. Endocrinol. 7:116. doi: 10.3389/fendo. 2016.00116

Helan, M., Aravamudan, B., Hartman, W. R., Thompson, M. A., Johnson, B. D., Pabelick, C. M., et al. (2014). BDNF secretion by human pulmonary artery endothelial cells in response to hypoxia. J. Mol. Cell Cardiol. 68, 89-97. doi: 10.1016/j.yjmcc.2014.01.006

Huang, E. J., and Reichardt, L. F. (2001). Neurotrophins: roles in neuronal development and function. Annu. Rev. Neurosci. 24, 677-736. doi: 10.1146/ annurev.neuro.24.1.677

Kayser, B., and Verges, S. (2013). Hypoxia, energy balance and obesity: from pathophysiological mechanisms to new treatment strategies. Obes. Rev. 14, 579-592. doi: 10.1111/obr.12034

Kohara, K., Kitamura, A., Morishima, M., and Tsumoto, T. (2001). Activitydependent transfer of brain-derived neurotrophic factor to postsynaptic neurons. Science 291, 2419-2423. doi: 10.1126/science.1057415

Krabbe, K. S., Nielsen, A. R., Krogh-Madsen, R., Plomgaard, P., Rasmussen, P., Erikstrup, C., et al. (2007). Brain-derived neurotrophic factor (BDNF) and type 2 diabetes. Diabetologia 50, 431-438. doi: 10.1007/s00125-006-0537-4
Kwon, D. H., Kim, B. S., Chang, H., Kim, Y. I., Jo, S. A., and Leem, Y. H. (2013). Exercise ameliorates cognition impairment due to restraint stress-induced oxidative insult and reduced BDNF level. Biochem. Biophys. Res. Commun. 434, 245-251. doi: 10.1016/j.bbrc.2013.02.111

Lasek-Bal, A., Jedrzejowska-Szypulka, H., Rozycka, J., Bal, W., Holecki, M., Dulawa, J., et al. (2015). Low concentration of BDNF in the acute phase of ischemic stroke as a factor in poor prognosis in terms of functional status of patients. Med. Sci. Monit. 21, 3900-3905. doi: 10.12659/MSM.895358

Laske, C., and Eschweiler, G. W. (2006). Brain-derived neurotrophic factor. vom nervenwachstumsfaktor zum plastizitätsmodulator bei kognitiven prozessen und psychischen erkrankungen. Der Nervenarzt 77, 523-537. doi: 10.1007/ s00115-005-1971-0

Laske, C., Stransky, E., Leyhe, T., Eschweiler, G. W., Maetzler, W., Wittorf, A., et al. (2007). BDNF serum and CSF concentrations in Alzheimer's disease, normal pressure hydrocephalus and healthy controls. J. Psychiatr. Res. 41, 387-394. doi: 10.1016/j.jpsychires.2006.01.014

Leschik, J., Eckenstaler, R., Nieweg, K., Lichtenecker, P., Brigadski, T., Gottmann, K., et al. (2013). Embryonic stem cells stably expressing BDNF-GFP exhibit a BDNF-release-dependent enhancement of neuronal differentiation. J. Cell Sci. 126, 5062-5073. doi: 10.1242/jcs.135384

Levada, O. A., Cherednichenko, N. V., Trailin, A. V., and Troyan, A. S. (2016). Plasma brain-derived neurotrophic factor as a biomarker for the main types of mild neurocognitive disorders and treatment efficacy: a preliminary study. Dis. Mark. 2016:4095723. doi: 10.1155/2016/4095723

Maass, A., Duzel, S., Brigadski, T., Goerke, M., Becke, A., Sobieray, U., et al. (2016). Relationships of peripheral IGF-1, VEGF and BDNF levels to exercise-related changes in memory, hippocampal perfusion and volumes in older adults. Neuroimage 131, 142-154. doi: 10.1016/j.neuroimage.2015.10.084

Malle, C., Bourrilhon, C., Quinette, P., Laisney, M., Eustache, F., and Pierard, C. (2016). Physiological and cognitive effects of acute normobaric hypoxia and modulations from oxygen breathing. Aerosp. Med. Hum. Perform. 87, 3-12. doi: 10.3357/AMHP.4335.2016

Matsuda, N., Lu, H., Fukata, Y., Noritake, J., Gao, H., Mukherjee, S., et al. (2009). Differential activity-dependent secretion of brain-derived neurotrophic factor from axon and dendrite. J. Neurosci. 29, 14185-14198. doi: 10.1523/ JNEUROSCI.1863-09.2009

Müller, P., Rehfeld, K., Schmicker, M., Hökelmann, A., Dordevic, M., Lessmann, V., et al. (2017a). Evolution of neuroplasticity in response to physical activity in old age: the case for dancing. Front. Aging Neurosci. 9:56. doi: 10.3389/fnagi.2017. 00056

Müller, P., Schmicker, M., and Müller, N. G. (2017b). Preventive strategies for dementia. Z. Gerontol. Geriatr. 50, 89-95. doi: 10.1007/s00391-017-1202-x

Navarrete-Opazo, A., and Mitchell, G. S. (2014). Therapeutic potential of intermittent hypoxia: a matter of dose. Am. J. Physiol. Regul. Integr. Comp. Physiol. 307, R1181-R1197. doi: 10.1152/aipregu.00208.2014

Neeper, S. A., Gomez-Pinilla, F., Choi, J., and Cotman, C. (1995). Exercise and brain neurotrophins. Nature 373:109. doi: 10.1038/373109a0

Nofuji, Y., Suwa, M., Sasaki, H., Ichimiya, A., Nishichi, R., and Kumagai, S. (2012). Different circulating brain-derived neurotrophic factor responses to acute exercise between physically active and sedentary subjects. J. Sports Sci. Med. 11, 83-88.

Pan, W., Banks, W. A., Fasold, M. B., Bluth, J., and Kastin, A. J. (1998). Transport of brain-derived neurotrophic factor across the blood-brain barrier. Neuropharmacology 37, 1553-1561. doi: 10.1016/S0028-3908(98)00141-5

Park, H., and Poo, M. M. (2013). Neurotrophin regulation of neural circuit development and function. Nat. Rev. Neurosci. 14, 7-23. doi: 10.1038/nrn3379

Parra, J., Cadefau, J. A., Rodas, G., Amigo, N., and Cusso, R. (2000). The distribution of rest periods affects performance and adaptations of energy metabolism induced by high-intensity training in human muscle. Acta Physiol. Scand. 169, 157-165. doi: 10.1046/j.1365-201x.2000.00730.x

Rasmussen, P., Brassard, P., Adser, H., Pedersen, M. V., Leick, L., Hart, E., et al. (2009). Evidence for a release of brain-derived neurotrophic factor from the brain during exercise. Exp. Physiol. 94, 1062-1069. doi: 10.1113/expphysiol. 2009.048512

Rehfeld, K., Lüders, A., Hökelmann, A., Lessmann, V., Kaufmann, J., Brigadski, T. et al. (2018). Dance training is superior to repetitive physical exercise in inducing brain plasticity in the elderly. PLoS One 13:e0196636. doi: 10.1371/ journal.pone. 0196636 
Rothman, S. M., and Mattson, M. P. (2013). Activity-dependent, stressresponsive BDNF signaling and the quest for optimal brain health and resilience throughout the lifespan. Neuroscience 239, 228-240. doi: 10.1016/j. neuroscience.2012.10.014

Ruscheweyh, R., Willemer, C., Kruger, K., Duning, T., Warnecke, T., Sommer, J., et al. (2011). Physical activity and memory functions: an interventional study. Neurobiol. Aging 32, 1304-1319. doi: 10.1016/j.neurobiolaging.2009.08.001

Schega, L., Peter, B., Brigadski, T., Lessmann, V., Isermann, B., Hamacher, D., et al. (2016). Effect of intermittent normobaric hypoxia on aerobic capacity and cognitive function in older people. J. Sci. Med. Sport 19, 941-945. doi: 10.1016/j.jsams.2016.02.012

Serebrovskaya, T. V., Manukhina, E. B., Smith, M. L., Downey, H. F., and Mallet, R. T. (2008). Intermittent hypoxia: cause of or therapy for systemic hypertension? Exp. Biol. Med. 233, 627-650. doi: 10.3181/0710-MR-267

Serebrovskaya, T. V., and Xi, L. (2016). Intermittent hypoxia training as nonpharmacologic therapy for cardiovascular diseases: practical analysis on methods and equipment. Exp. Biol. Med. 241, 1708-1723. doi: 10.1177/ 1535370216657614

Sharp, F. R., and Bernaudin, M. (2004). HIF1 and oxygen sensing in the brain. Nat. Rev. Neurosci. 5, 437-448. doi: 10.1038/nrn1408

Smith, M. A. (1996). Hippocampal vulnerability to stress and aging: possible role of neurotrophic factors. Behav. Brain Res. 78, 25-36. doi: 10.1016/0166-4328(95) 00220-0

Smith, M. A., and Cizza, G. (1996). Stress-induced changes in brain-derived neurotrophic factor expression are attenuated in aged Fischer $344 / \mathrm{N}$ rats. Neurobiol. Aging 17, 859-864. doi: 10.1016/S0197-4580(96)00066-8

Sokoloff, P., Leriche, L., and Diaz, J. (2004). Brain-derived neurotrophic factor (BDNF) bridges the gap between dopamine and glutamate dysfunctions in schizophrenia. Behav. Pharmacol. 15, A4-A4. doi: 10.1097/00008877200409000-00030

Tsai, Y. W., Yang, Y. R., Wang, P. S., and Wang, R. Y. (2011). Intermittent hypoxia after transient focal ischemia induces hippocampal neurogenesis and c-Fos expression and reverses spatial memory deficits in rats. PLoS One 6:e24001. doi: 10.1371/journal.pone.0024001

Uysal, N., Kiray, M., Sisman, A. R., Camsari, U. M., Gencoglu, C., Baykara, B., et al. (2015). Effects of voluntary and involuntary exercise on cognitive functions, and VEGF and BDNF levels in adolescent rats. Biotech. Histochem. 90, 55-68. doi: 10.3109/10520295.2014.946968
Vermehren-Schmaedick, A., Jenkins, V. K., Knopp, S. J., Balkowiec, A., and Bissonnette, J. M. (2012). Acute intermittent hypoxia-induced expression of brain-derived neurotrophic factor is disrupted in the brainstem of methyl-CpGbinding protein 2 null mice. Neuroscience 206, 1-6. doi: 10.1016/j.neuroscience. 2012.01.017

Viscor, G., Torrella, J. R., Corral, L., Ricart, A., Javierre, C., Pages, T., et al. (2018). Physiological and biological responses to short-term intermittent hypobaric hypoxia exposure: from sports and mountain medicine to new biomedical applications. Front. Physiol. 9:814. doi: 10.3389/fphys.2018. 00814

Wang, W. H., He, G. P., Xiao, X. P., Gu, C., and Chen, H. Y. (2012). Relationship between brain-derived neurotrophic factor and cognitive function of obstructive sleep apnea/hypopnea syndrome patients. Asian Pac. J. Trop. Med. 5, 906-910. doi: 10.1016/S1995-7645(12)60169-2

Wang, Y., Chai, Y., He, X., Ai, L., Sun, X., Huang, Y., et al. (2017). Intermittent hypoxia simulating obstructive sleep apnea causes pulmonary inflammation and activates the Nrf2/HO-1 pathway. Exp. Ther. Med. 14, 3463-3470. doi: $10.3892 /$ etm.2017.4971

Wiener, C. M., Booth, G., and Semenza, G. L. (1996). In vivo expression of mRNAs encoding hypoxia-inducible factor 1. Biochem. Biophys. Res. Commun. 225, 485-488. doi: 10.1006/bbrc.1996.1199

Yan, X. (2014). Cognitive impairments at high altitudes and adaptation. High Alt. Med. Biol. 15, 141-145. doi: 10.1089/ham.2014.1009

Zhu, L. L., Zhao, T., Li, H. S., Zhao, H., Wu, L. Y., Ding, A. S., et al. (2005). Neurogenesis in the adult rat brain after intermittent hypoxia. Brain Res. 1055, 1-6. doi: 10.1016/j.brainres.2005.04.075

Conflict of Interest Statement: The authors declare that the research was conducted in the absence of any commercial or financial relationships that could be construed as a potential conflict of interest.

Copyright (c) 2018 Becke, Müller, Dordevic, Lessmann, Brigadski and Müller. This is an open-access article distributed under the terms of the Creative Commons Attribution License (CC BY). The use, distribution or reproduction in other forums is permitted, provided the original author(s) and the copyright owner(s) are credited and that the original publication in this journal is cited, in accordance with accepted academic practice. No use, distribution or reproduction is permitted which does not comply with these terms. 